\title{
A Method of Identifying Improvement Factors for Tablet Services
}

\author{
Motoi Iwashita *
}

\begin{abstract}
Upon the introduction of goods/services into the market, every company observes customer reaction. Thereafter, the goods/services are improved based on customer feedback. Customer satisfaction is one of the prominent and effective indicators of customer retention. Identifying the areas of improvement for goods/services is an essential issue for every company, when applying customer satisfaction. This is especially important given the rapid and prolific production of many technologies in the field of information and communication technology (ICT). Moreover, many players including network carriers, vendors, and content providers, are concerned with the services related to ICT. Therefore, the improvement factors contain the different viewpoints of several players. For these reasons, a transitional evaluation approach is needed in addition to the conventional evaluation, which merely compares satisfaction/dissatisfaction scores. This paper focuses on tablet PCs and their services, which have been broadly diffused. Following this, a transitional evaluation approach for identifying long-term improvement factors of tablet PCs and their services is proposed.
\end{abstract}

Keywords: Customer satisfaction, data mining, ICT services, service diffusion, service management.

\section{Introduction}

Every company invests considerable efforts in maintaining their customers over time, especially after the introduction of new goods/services in the market. They carefully monitor customer reaction and pursue improvements of goods/services based on customer feedback. A Customer satisfaction (CS) is widely accepted as an indicator that enables the identification of improvement factors for customer retention. The evaluation of CS and identification of factors of improvement is generally conducted through the questionnaire results obtained from consumers. Some companies employ social networking services (SNS) such as Twitter and Facebook in addition to the questionnaire. A simple way to identify the factors that may improve CS is to select those factors that have received high dissatisfaction scores. Such scores are available even for the goods/services that are provided by only one company. However, these dissatisfaction factors may apply to not only equipment, but also network and content in the ICT field, given its remarkable technological development, competitors, and contextual environment.

\footnotetext{
* Chiba Institute of Technology, Chiba, Japan
} 
Therefore, it is expected for ICT related companies to identify adequately the changes in customer perceptions or underlying dissatisfaction factors related to ICT services.

For instance, the tablet PC has been gaining wide acceptance since its inception in 2010 with nearly 5 million tablet PCs shipped to Japan in 2012. The development of many types of operating systems (OSs) has increased the choices available to users. The tablet PC may have great potential for use by the elderly due to its ease of operation and screen visibility compared with the smartphone. Moreover, the tablet PC is superior to a standard PC in terms of the business benefit of having a short start-up time. The dissatisfaction score may be related to usage requirements and a variety of issues stemming from demand growth, e.g., the absence of one-segment broadcasting is one of the highest dissatisfaction causing factors of the tablet PC. "One-segment broadcasting" is a digital, terrestrial broadcasting service for mobile phones in Japan; tablet PCs are not yet equipped with a one-segment broadcasting function. However, the associated dissatisfaction decreased from 2011 to 2012, which may indicate that the necessity of one-segment broadcasting has diminished, potentially due to the availability of alternative means of video watching including the frequent use of free video distribution platforms such as YouTube.

It is necessary to derive the relationship between platform development vendors, network carriers, and application providers for the diffusion of tablet PCs.

This paper focuses on the tablet PC and its services. Several evaluation methods for identifying improvement factors are discussed based on the perspective of satisfaction and dissatisfaction. In addition, the transitional evaluation method is proposed for identifying long-term improvement factors.

Section 2 describes the issues related to CS as an indicator and its application to ICT services. Section 3 is devoted to the framework of service diffusion, especially for ICT services. Tablet features are introduced in Section 4. Data analysis and considerations are discussed in Section 5.

\section{Related Studies}

The concept of CS was proposed in [1]. It is a way of thinking based on the demands and tastes of consumers, as opposed to producers. Paying attention to consumer comments is the best approach to identify the improvement factors of goods/services for higher CS. Therefore, it is necessary to capture consumers' demands adequately in order to ensure strong consumer loyalty. The target of an individual company is mainly the core user and the aim is to understand core user satisfaction at the time of use. While CS is an effective indicator of core customer retention, it is difficult to understand/target potential consumers and to spread the use of their goods. The JCSI (Japan Customer Satisfaction Index) provides one approach to evaluating CS as expounded in [2]. The JCSI covers all customers with usage experience in order to understand the overall customer perceptions and to note the features of importance to them. Company evaluation may be conducted based on the core and light customer experience at a certain time. The results are used by the company for management targets and policy development, but cannot be used to identify improvement factors. The strategy of a company significantly depends on the target customer segment such as innovator, early adopter, early majority, late majority, and laggard [3]. It should also carefully consider the chasm that exists between early adopters and early majority in order to increase the penetration of goods/services [4].

The telecommunications field comprises several players such as network carriers, equipment vendors, and information providers. This makes it difficult to understand and classify whether the obtained CS results are related to issues with the network carrier, equipment vendor, or in- 
formation provider. Reference [5] investigates the characteristics of CS with mobile services by decision tree. Broadband access, such as fiber-to-the-home (FTTH), and mobile services are investigated from the viewpoint of user choice behavior [6], [7]. The relationship between CS and switching effect is studied in the context of mobile services [8], [9]. Moreover, customer services and price fairness are described to be important factors for CS in the field of telecommunications [10]. These results have illuminated past user intentions, but failed to provide guidelines or methods for identifying the factors that companies should focus on in the future. Moreover, the sheer number and various types of customers that use ICT services further complicate the adequate identification of improvement factors.

\section{Service Diffusion Framework}

A strategic framework to diffuse the tablet PC and its services should be constructed, as shown in Figure 1. The service diffusion process involves three types of activities: "service optimization," "sales promotion," and "Sale." Firstly, a company collects and understands customer requirements including service cost, equipment weight, and security requirements through questionnaire surveys or SNS. Thereafter, the service is improved in terms of price, operability, value-added services, and others such that the service is optimized. Hence, the main activities involved in service optimization are investment in facilities and understanding of customer requirements. The sales promotion plan includes advertisements, price reduction campaigns, ample supply of commemorative goods, and so on. Finally, the sale is completed and the results are feedback.

The basic concept of CS may be explained as follows. Customer loyalty generally depends on the relationship among three factors: price, customer expectation (CE), and CS. Price is decided at the phase of service optimization, customer expectations of goods/services are set based on sales promotions, and the customer satisfies these expectations upon obtaining the goods/services, as shown in Figure 1. The good/service is considered to be supported by a good strategy when CS is higher than CE. Loyalty decreases when this relationship is reversed, although the damage would be relatively small as long as CS is greater than price. However, customer retention will be difficult if CS is lower than price. Therefore, CS increases when CS is larger than CE; thereby, the relationship between CE and CS is important.

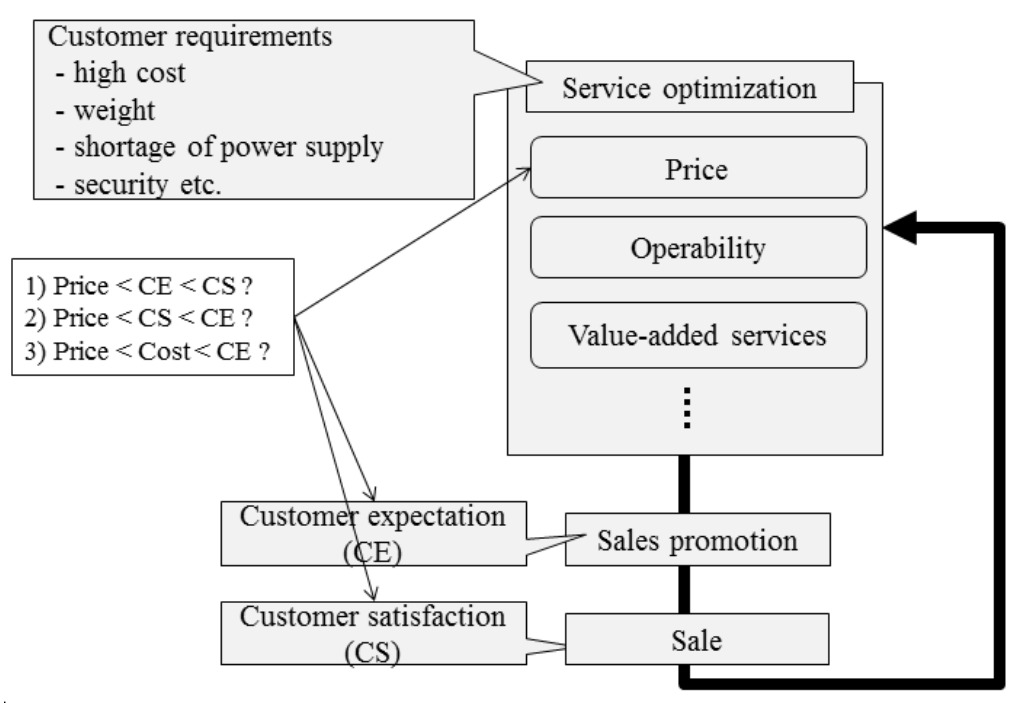

Figure 1: Framework of tablet service diffusion 


\section{Tablet PC Features}

Tablet PCs generally operate on three OSs, namely iOS, Android, and Windows, which have several distinguishing features, including harddisk (HDD) capacity, price, pixels, weight, and waterproof status. We investigated these data in 2013, as shown in Table 1. Several types of tablet PCs with Android and Windows OS exist, and potential consumers are able to select a tablet from a variety of sizes and prices. The HDD capacity is large for iOS and Windows. The price variation of Windows tablets is the largest among the three as their target users are not only consumers but also businesses.

Apple Inc. (iOS) was the first to introduce tablets in 2010. Over the years, Android has gradually increased in production such that both these OSs claimed almost equal sales volumes as of 2012. As Windows entered the tablet PC market only in 2011, its volume remains small currently, as shown in Figure 2.

Table 1: Tablet features for different OSs

\begin{tabular}{llll}
\hline Features & iOS (28)* & Android (126) & Windows (152) \\
\hline \hline Size (inch) & $8-10$ & $5.5-10$ & $8-21.5$ \\
HDD (GB) & $16-128$ & $4-64$ & $32-256$ \\
Price (1000 yen) & $29.7-95.7$ & $6.8-122.4$ & $38.1-262.9$ \\
Pixels (million) & 1.2 & $0.3-2$ & $0.92-2$ \\
Weight (g) & $308-652$ & $210-765$ & $350-5000$ \\
Waterproof & 0 & 7 & 23 \\
(number of prod- & & & \\
ucts) & & & \\
\hline
\end{tabular}

* Bracket means the number of products

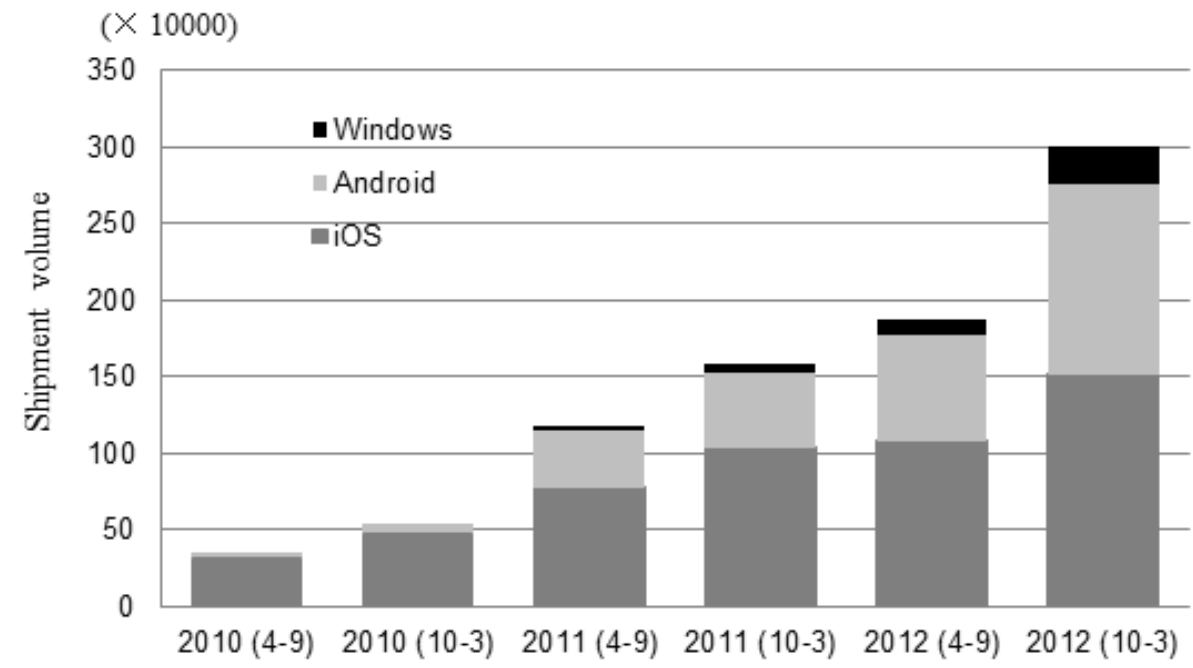

Figure 2: Tablet shipment volume by OS in Japan 
Tablet PCs are generally connected to the internet, and hence, wireless network services are a key enabling feature. WiMAX, provided by UQ Communications Inc., has served about four million customers over three years since June 2010. However, its rate of increase has slowed recently. LTE, provided by NTT Docomo, has covered 16 million customers since December 2010. Each of these broadband services has served as an engine to support explosive tablet PC use as shown in Figure 3.

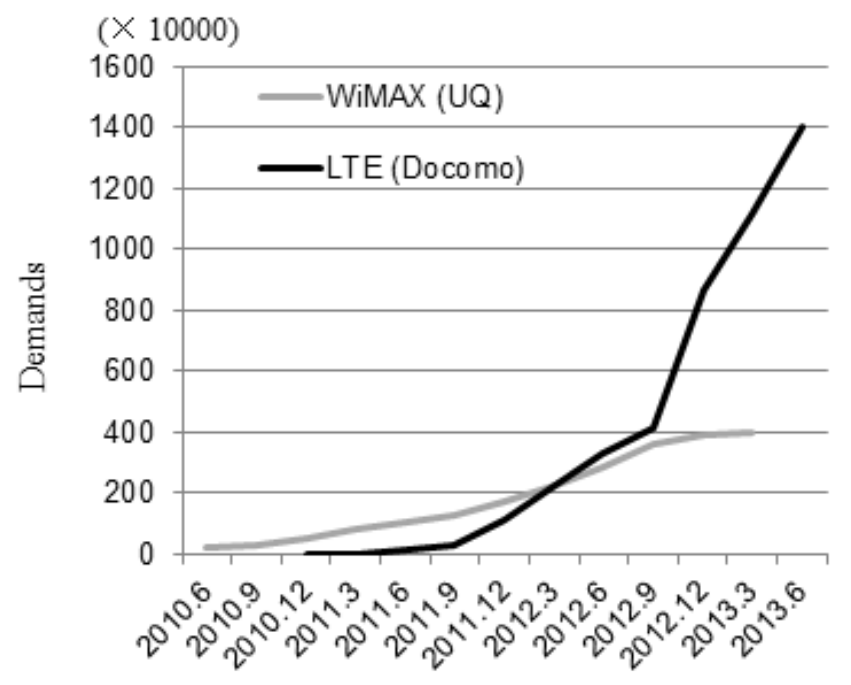

Figure 3: Wireless broadband demands

\section{Analysis and Considerations}

\subsection{Questionnaire Results}

Table 2 shows the results of the data collected through the questionnaire regarding the tablet PC and its services in 2011 and 2012 (Impress R\&D, 2012) [11]. Given that CE and CS are relative values, the points of dissatisfaction obtained by the questionnaire may be indicated by the factors with negative differences between CS and CE. On the other hand, the points of satisfaction may be indicated by positive differences.

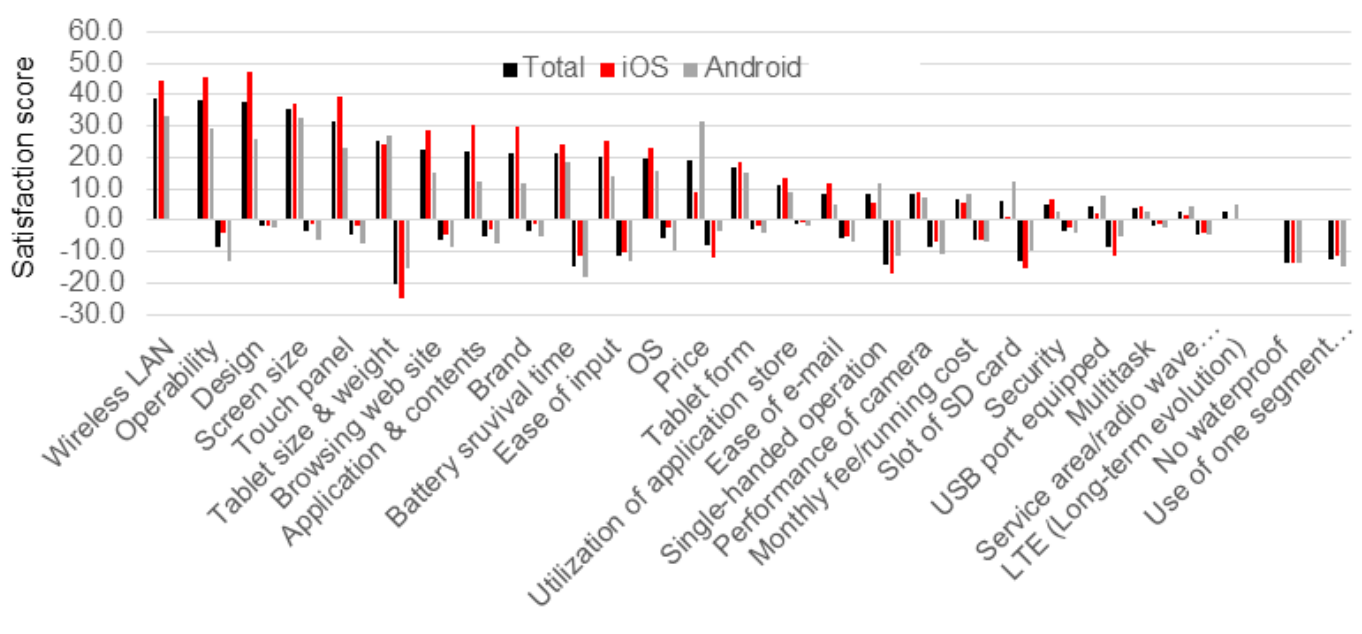

Figure 4: Satisfaction scores by OS 
Table 2: Questionnaire Results based on “Total” Scores [11]

\begin{tabular}{|c|c|c|c|c|}
\hline \multirow[t]{2}{*}{ Items } & \multicolumn{2}{|c|}{ Satisfied } & \multicolumn{2}{|c|}{ Dissatisfied } \\
\hline & 2011 & 2012 & 2011 & 2012 \\
\hline Wireless LAN & 38.1 & 38.9 & 0.0 & 0.0 \\
\hline Operability & 43.8 & 38.1 & 5.1 & 8.3 \\
\hline Design & 44.8 & 37.5 & 1.2 & 1.8 \\
\hline Screen size & 33.2 & 35.1 & 2.3 & 3.7 \\
\hline Touch panel & 33.6 & 31.6 & 3.0 & 4.4 \\
\hline Tablet size \& weight & 22.3 & 25.4 & 17.8 & 20.5 \\
\hline Browsing web site & 21.3 & 22.4 & 4.8 & 6.4 \\
\hline Application \& content & 29.3 & 22.1 & 2.0 & 5.0 \\
\hline Brand & 20.1 & 21.4 & 1.4 & 3.3 \\
\hline Battery survival time & 22.6 & 21.4 & 15.7 & 14.6 \\
\hline Ease of input & 20.7 & 20.0 & 10.0 & 11.5 \\
\hline OS & 18.2 & 19.5 & 2.1 & 5.9 \\
\hline Price & 13.0 & 19.2 & 11.6 & 8.0 \\
\hline Tablet form & 18.1 & 17.1 & 2.0 & 2.9 \\
\hline Utilization of app-store & 9.9 & 11.3 & 1.2 & 1.0 \\
\hline Ease of e-mail & 8.0 & 8.5 & 4.7 & 5.9 \\
\hline Single-handed operation & 5.7 & 8.5 & 18.0 & 14.3 \\
\hline Performance of camera & 5.2 & 8.2 & 10.8 & 8.7 \\
\hline Monthly fee/running cost & 8.5 & 6.8 & 5.8 & 6.4 \\
\hline SD card slot & 5.1 & 6.2 & 12.4 & 12.8 \\
\hline Security & 4.0 & 5.0 & 5.0 & 3.2 \\
\hline USB port equipped & 4.1 & 4.6 & 10.5 & 8.6 \\
\hline Multitasking & 3.1 & 3.7 & 1.7 & 1.7 \\
\hline Service area/radio wave condition & 0.0 & 3.0 & 0.0 & 4.4 \\
\hline LTE (Long-term evolution) & 0.0 & 2.7 & 0.0 & 0.0 \\
\hline No waterproof & 0.0 & 0.0 & 18.7 & 13.7 \\
\hline Use of one segment broadcast & 0.0 & 0.0 & 13.2 & 12.7 \\
\hline
\end{tabular}

\subsection{Satisfaction and Dissatisfaction Score Perspectives}

Firstly, satisfaction/dissatisfaction with various factors by OS is analyzed, as presented in Figure 
4. The factors with the three highest satisfaction scores are "Design," "Operability," and "Wireless LAN" for iOS, and "Screen size," "Wireless LAN", and "Price” for Android. The dissatisfaction score is highest for "Tablet size \& weight" in the case of iOS, while it is highest for "Battery survival time" in the case of Android. Therefore, the factor for improvement in CS highly depends on the type of OS. Although the top factors are not the same across the two OSs, the scores tend to be similar. Hence, hereafter, we focus on the total scores to identify representative improvement factors.

The factors with the three highest dissatisfaction scores in the total category in 2012 are "Tablet size \& weight," "Battery survival time," and "Single-handed operation" according to Table 2 and Figure 4. Although these factors received high dissatisfaction scores, they also received high points for satisfaction. This may be because users who are already accustomed to using a particular tablet PC may be favorably disposed, whereas new users are inconvenienced. The trend from 2011 to 2012 shows no change in the satisfaction scores of these factors, while the dissatisfaction score decreases for these factors except for "Tablet size \& weight." Given that users perform a significant number of tasks, including SNS communications, using their tablet PC, the decreased dissatisfaction with "Battery survival time" and "Single-handed operation" may be a result of user accustomization in addition to device improvement. Number of customers is considered to increase rapidly because of the tablet shipment volume in 2012 doubled compared with that in 2011 as described in the previous section. Therefore, both satisfaction and dissatisfaction increase for "Tablet size \& weight," potentially due to the variety of tablet types available in the market in response to the increasing demand. As this factor cannot be controlled through user effort, it is necessary to continuously monitor how consumers use their tablet PCs and how these fit into their lifestyle.

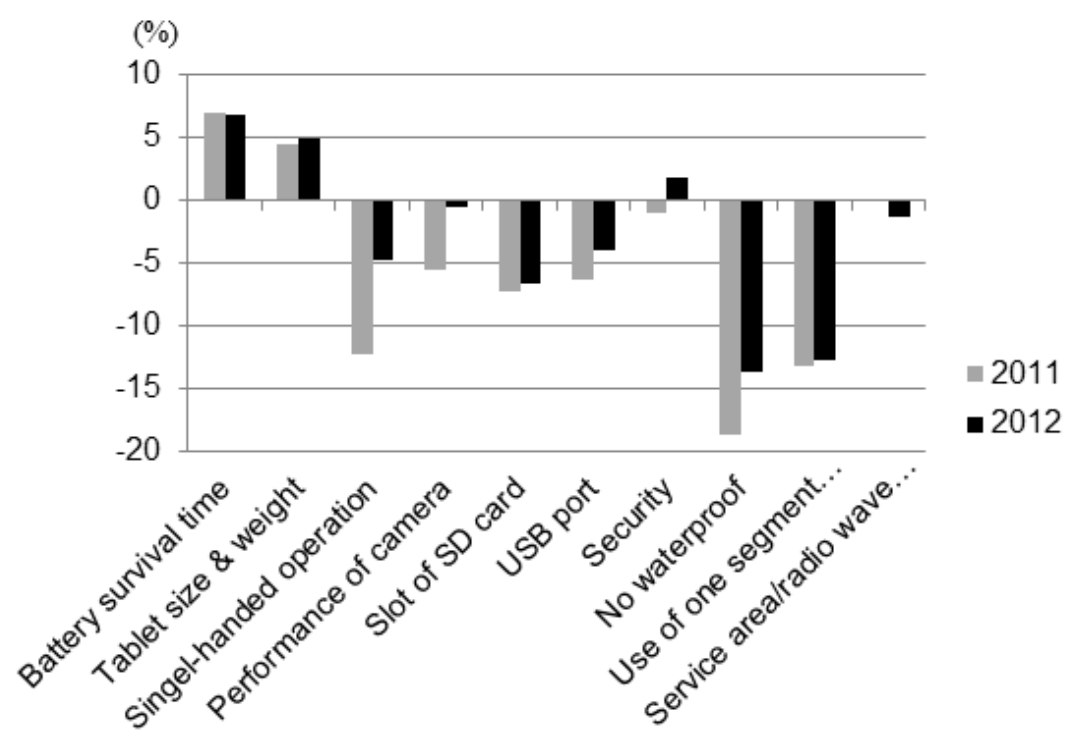

Figure 5: Difference of satisfaction and dissatisfaction per year

\subsection{Difference between Satisfaction and Dissatisfaction}

Next, the difference between satisfaction and dissatisfaction is investigated, as shown in Figure 5. In 2011 and 2012, the difference between satisfaction and dissatisfaction was positive for "Bat- 
tery survival time" and "Tablet size \& weight." In the case of "Single-handed operation," the difference was negative but decreased from 2011 to 2012. Therefore, these factors may be considered not very critical from the viewpoint of difference score.

The difference of "Service area/radio wave condition" in 2011 did not appear this is because no data are collected for this item. However, the difference in 2012 appeared as the negative value. This result is considered to depend on network facility deployment. Since many customers use tablet PC year by year, such facility limitation would be a bottleneck for the service penetration. It is necessary to deploy facilities for many areas by the network service providers in a planned manner.

For "No waterproof" and "Use of one-segment broadcasting," the difference was a large negative value. Since the difference decreased from 2011 to 2012, it could be assumed that people got accustomed to using tablet PCs without these functions. In the same manner, other factors with negative values were observed to have a smaller negative difference in 2012 compared to 2011. It may be assumed that some improvements have been made for several factors.

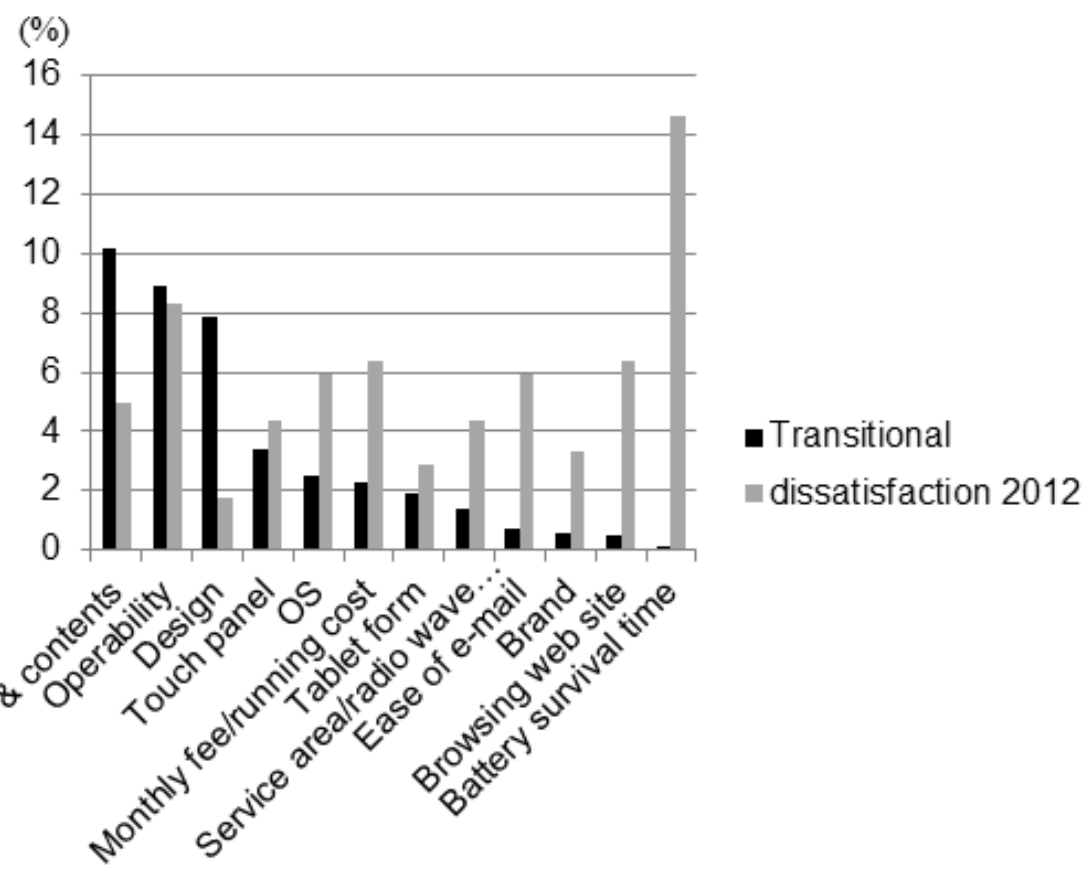

Figure 6: Algorithm and result

\subsection{Transitional Approach}

In considering the change in customer perceptions and the underlying dissatisfaction factors, the difference between the satisfaction and dissatisfaction trends is important. Here, the values are defined as follows:

1. Let $A$ be the difference in satisfaction scores from 2011 to 2012.

2. Let $B$ be the difference in dissatisfaction scores from 2011 to 2012.

The results based on these definitions are shown in Figure 6 and are based on the assumption that an increase/decrease in satisfaction is independent of an increase/decrease in dissatisfaction. The bar chart indicates the transitional dissatisfaction point, $D$, which is based on the difference between the satisfaction and dissatisfaction trends as follows:

Step 1: If $A>0$ and $B>0$, 
Step 1-1: If $\mathrm{A}>\mathrm{B}$, then $\mathrm{D}=0$.

Step 1-2: If $\mathrm{A}<\mathrm{B}$, then $\mathrm{D}=\mathrm{B}-\mathrm{A}$.

Step 2: If $\mathrm{A}>0$ and $\mathrm{B}<0$, then $\mathrm{D}=0$.

Step 3: If $\mathrm{A}<0$ and $\mathrm{B}>0$, then $\mathrm{D}=|\mathrm{A}|+|\mathrm{B}|$.

Step 4: If $A<0$ and $B<0$,

Step 4-1: If $|\mathrm{A}|>|\mathrm{B}|$, then $\mathrm{D}=|\mathrm{A}|-|\mathrm{B}|$.

Step 4-2: If $|\mathrm{A}|<|\mathrm{B}|$, then $\mathrm{D}=0$.

"Application \& contents," "Operability,” and "Design” were the top three transitional factors. The values of "Tablet size \& weight," "Buttery survival time," and "Single-handed operation" are almost 0 . The transitional approach yields different factors compared to the other approaches described in Sections 5.2 and 5.3. It is easy to understand that more applications and contents are provided, more and longer customers use tablet services. Since a variety of users including children and the elderly increase, user-friendliness such as easy configuration is essential for penetrating the service universally. A mobile gadget such as smartphone and wearable equipment becomes a nifty gadget especially for young adult these days. Therefore, catching up stylish design is also essential. Therefore, this transitional approach may be considered to reveal potential and essential user perceptions. Furthermore, it can be said that improving these three items is a key issue for providing tablet services for a long time. Therefore, mutual cooperation with providers is essential.

\section{Conclusion}

Customer satisfaction is essential for customer retention. Given the rapid development of ICT in every field, it is ineffective to focus only on improvement factors that satisfy/dissatisfy users at that time. It is also important to identify adequately any changes in customer perceptions or underlying dissatisfaction factors. For this reason, a time-series analysis considering a change in circumstances is important for goods/services related to ICT. This paper discusses a transitional approach to identify improvement factors for the tablet PC and its services.

Future studies should focus on analyzing the relationships among factors, generations, and genders in order to characterize further the tablet PC and its services. This may also be necessary in order to evaluate the validity of the proposed approach with different types of ICT services.

\section{References}

[1] C. Denove and J. D. Power IV, Satisfaction (in Japanese), Diamondsya, 2006.

[2] C. Minami and K. Ogawa, "Model development of Japanese Customer Satisfaction Index (JCSI) and the theoretical basis,” Japan Marketing Journal, vol. 30, no. 1, 2010, pp. 4-19.

[3] E. Rogers, Diffusion of innovation: Fifth edition (in Japanese), Shoeisya, 2007.

[4] G. A. Moore, Crossing the chasm: Marketing and selling high-tech goods to mainstream 
customers, Harper Business, 1991.

[5] J. Choi, H. Seol, S. LEE, H. Cho and Y. Park, "Customer satisfaction factors of mobile commerce in Korea,” Internet Research, vol. 18, no. 3, 2008, pp. 313-335.

[6] T. Kurosawa, H. Kawano, M. Iwashita, S. Shimogawa, S. Kouno and A. Inoue, ”Econometric Approach for Broadband Market in Japan,” Knowledge-Based and Intelligent Information and Engineering Systems, LNAI 6278, Springer, 2010, pp. 225-234.

[7] A. Inoue, Y. Takano, T. Kurosawa, M. Iwashita and K. Nishimatsu, "Mobile-Carrier Choice Modeling Under Competitive Conditions,” Journal of Information Processing, vol. 20, no. 3, 2012, pp. 585-591.

[8] J. Lee, J. Lee and L. Feick, "The impact of switching cost on the customer satisfaction-loyalty link: mobile phone service in Franc,” Journal of Services Marketing, MCB UP Ltd., vol. 15, no. 1 , pp. 35-48.

[9] M. K. Kim, H. C. Park and D. H. Jeong, "The effects of customer satisfaction and switching barrier on customer loyalty in Korean mobile telecommunication services," Telecommunications Policy, Elsevier, vol. 28, no. 2, 2004, pp. 145-159.

[10] M. Hanif, S. Hafeez and A. Riaz, "Factors affecting Customer Satisfaction,” International Research Journal of Finance and Economics, EuroJournals Publishing Inc., Issue 60, 2010, pp. 44-52.

[11] An Impress Group Company, Internet Media Labs., Tablet Research Report 2013, Impress R\&D, 2012. 